\title{
Exploring Associations Between Oral Health and Frailty in Community- Dwelling Older People
}

\author{
R.C. Castrejón-Pérez
}

Instituto Nacional de Geriatría, National Institutes of Health, Health Ministry, Mexico

Corresponding Author: R.C. Castrejón-Pérez, Instituto Nacional de Geriatría, National Institutes of Health, Health Ministry, Mexico, rc.castrejon.perez@gmail.com, rcastrejon@inger.gob.mx

Dear Editor,

$\mathrm{T}$ he studies exploring the association between oral conditions and Frailty status are increasing in number, and many manuscripts have been published during the last couple of years. Even when Everaars et al. (1) manuscript is cross-sectional, it contributes to the knowledge by confirming the association between oral conditions and Frailty despite the selected strategy for measuring Frailty since authors added the interview Groningen Frailty Index and the Frailty Index (computed with data extracted from the Electronic Medical Record) to the most frequently used Frailty Phenotype and Kihon checklist (2).

The reviews by Hakeem et al. (2) and Torres et al. (3) summarised the oral characteristics (objective and subjective) associated with Frailty among older persons. However, oral problems occur simultaneously and interact with each other, resulting in a complex of oral conditions with a potentially adverse effect on oral functioning and compromising diet quality and nutritional status. Therefore, Everaars et al. add a diamond in the rock by referring to Oral Complications, implying the cumulative characteristic of oral conditions and the interaction between oral conditions and general conditions (such as sarcopenia) as suggested by Azzolino et al. (4)

The first measurement considering a cluster of oral conditions or oral complications is the Oral Frailty measurement, which in recent years is gaining popularity. It was first introduced in 2017 (5) and operationalized in 2018 (6) by combining the measurement of six oral characteristics (the number of teeth, chewing ability, articulatory motor skill for «ta,» tongue pressure, self-reported difficulty in eating tough foods, and self-reported difficulty in swallowing) into a single measurement. This combination resulted in a novel evaluation for Multidimensional Oral Function considering three dimensions (clinical characteristics, objective functional measurement, and self-reported), which additionally demonstrated predictability for Physical Frailty, Sarcopenia, Disability, and Mortality. (6)
Nutrition is among the most recognized factors associated with Frailty's development, and it seems to be the main link between oral conditions and Frailty. The evidence linking oral conditions and Frailty is increasing with cross-sectional and prospective approaches. The main solo oral conditions associated with Frailty are the number of teeth, which by itself is associated with chewing difficulties and the need for removable dental prostheses, among others. Several interventions aiming to treat and manage the older persons' Frailty status have been published focusing on nutritional interventions and exercise; however, none included an oral component (education or treatment) as part of the intervention.

Everaars et al.'s (1) findings are consistent with previous studies on the association between oral conditions and older persons' Frailty status under cross-sectional and prospective approaches. Therefore, it is relevant to consider older people's oral conditions to design and plan person-centered and populational-centered interventions and to design the older persons' frailty treatment or management. It is also relevant to investigate the potential effect of oral interventions on older persons' Frailty status.

Conflicts of Interest: I declare that I have no conflict of interest.

\section{References}

1. Everaars B, Jerković-Ćosić K, Bleijenberg N, de Wit NJ, van der Heijden G. Exploring Associations between Oral Health and Frailty in Community-Dwelling Older People. J Frailty Aging. 2021;10(1):56-62.

2. Hakeem FF, Bernabe E, Sabbah W. Association between oral health and Frailty: A systematic review of longitudinal studies. Gerodontology. 2019.

3. Torres LH, Tellez M, Hilgert JB, Hugo FN, de Sousa MD, Ismail AI. Frailty, Frailty Components, and Oral Health: A Systematic Review. J Am Geriatr Soc. 2015;63(12):2555-62.

4. Azzolino D, Passarelli PC, De Angelis P, Piccirillo GB, D'Addona A, Cesari M. Poor Oral Health as a Determinant of Malnutrition and Sarcopenia. Nutrients. 2019;11(12).

5. Kera T, Kawai H, Yoshida H, Hirano H, Kojima M, Fujiwara Y, et al. Classification of Frailty using the Kihon checklist: A cluster analysis of older adults in urban areas. Geriatr Gerontol Int. 2017;17(1):69-77.

6. Tanaka T, Takahashi K, Hirano H, Kikutani T, Watanabe Y, Ohara Y, et al. Oral Frailty as a Risk Factor for Physical Frailty and Mortality in Community-Dwelling Elderly. J Gerontol A Biol Sci Med Sci. 2018;73(12):1661-7.

How to cite this article: R.C. Castrejón-Pérez. Exploring Associations Between Oral Health and Frailty in Community-Dwelling Older People. J Frailty Aging 2021;10(4)367; http://dx.doi.org/10.14283/jfa.2021.10 